\title{
ESTADO DA ARTE DA RELAÇÃO ENTRE IRISINA E COGNIÇÃO
}

\author{
STATE OF THE ART OF THE RELATION BETWEEN IRISIN AND \\ COGNITION
}

\author{
Antonio Vituriano de Abreu Neto ${ }^{1}$ \\ Aracele Gonçalves Vieira ${ }^{2}$ \\ Renata Lívia S. F. Moreira ${ }^{3}$ \\ Paulo Antônio Farias Lucena ${ }^{4}$
}

\begin{abstract}
RESUMO: OBJETIVO: O objetivo deste estudo foi reunir os achados de diferentes pesquisas a respeito, a fim de conhecer a relação entre irisina e cognição. MÉTODO: Este estudo consistiu em verificar o estado da arte acerca dos estudos sobre irisina e cognição. RESULTADOS: Inicialmente, introduziu-se a estratégia de busca "irisin or myokine and cognition" nas bases PubMed e Medline. No total, foram encontrados 762 artigos, sendo dez do PubMed e 752 do Medline. Em seguida, realizou -se uma segunda análise, excluindo as publicações repetidas, retiveram-se
\end{abstract}

\footnotetext{
${ }^{1}$ Graduando em Medicina pela Faculdade - FSM.

2 Mestre em Saúde e Sociedade pela Universidade Estadual do Rio Grande do Norte (2017); Especialista em docência do Ensino Superior (2017); Especialista em Programa Saúde da Família (2007); Possui Graduação em Fisioterapia pelo Centro Universitário de João Pessoa (2003). Atualmente, faz parte do corpo docente da Faculdade Santa Maria (Fisioterapia e Medicina), Tutora e conteudista da Educação à Distância (Disciplina Metodologia do trabalho científico), compõe a CPA (Comissão Própria de Avaliação); Tutora da Residência Multiprofissional em Saúde Coletiva; Tem experiência nas áreas Atenção Primária à Saúde, Epidemiologia, Anatomia e Saúde do idoso.

3 Doutora pela Faculdade de Ciências Médicas Santa Casa - FCMSCSP. Mestre em Enfermagem pela Universidade Federal da Paraíba (2013). Especialista em Saúde Pública pela Faculdade de Ciências Sociais e Aplicadas (2008). Graduada em Enfermagem pela Universidade Federal da Paraíba (2003). Docente da Faculdade Santa Maria; Membro do Grupo de Estudos e Pesquisas sobre Masculinidades e Saúde. Tem experiência na área de Enfermagem Saúde Pública e atua principalmente nos seguintes temas: educação em enfermagem, saúde sexual e saúde reprodutiva, educação em saúde, saúde da mulher, saúde do homem e atenção básica.

${ }^{4}$ Graduado em Medicina pelo Centro Universitário de Volta Redonda (2006). Doutorando em Ciências da Saúde - Medicina I - Faculdade de Medicina do ABC-SP, com mestrado em Ciências da Saúde com área de atuação em Neurologia pela Faculdade de Ciências Médicas da Universidade de Pernambuco (2014). Tem residência médica em neurologia no Hospital Universitário Oswaldo Cruz FCM-UPE (2012). Atualmente, atua como professor de Neurologia e Semiologia Neurológica na Faculdade de Medicina Nova Esperança - FAMENE e Faculdade Santa Maria - FSM - Cajazeiras-PB. Tem experiência em Gestão Acadêmica, hospitalar e empresarial, com MBA nas áreas. Experiência em metodologias ativas de ensino desde 2007.
} 
688 artigos (90,29\%). Foram retidos 26 artigos (3,41\%). Dentre os principais motivos para eliminação estiveram: se tratarem de estudos empíricos; não abordavam a relação entre miocina e cognição. CONCLUSÃO: Em suma, esta revisão sistemática demonstrou a relevância de estudar a relação entre irisina e cognição, e que essa ainda carece de mais estudos científicos que venham a embasar e estabelecer os limites existentes entre elas.

DESCRITORES: Irisina; Miocinas; Cognição.

ABSTRACT: OBJECTIVE: The objective of this study was to gather the findings from different researches related to it, in order to know the relationship between irisin and cognition. METHOD: This study consisted of checking the state of the art about irisin and cognition studies. RESULTS: Initially, the search strategy 'irisin or myokine and cognition' was introduced in the PubMed and Medline databases (searching websites fed from National Library of Medice). As total, 762 articles were found, ten from PubMed and 752 from Medline. As a following, a second analysis was performed, excluding repeated publications, withholding 688 articles (90.29\%). Twenty-six articles were retained (3.41\%). Among the main reasons for elimination there were such as: because they are empirical studies; they did not address the relationship between myokine and cognition. CONCLUSION: In summary, this systematic review demonstrated the relevance of studying the relation between irisin and cognition, and that it still holds needs for further scientific studies to support and establish the boundaries between them.

DESCRIPTORS: Irisin; Myokines; Cognition. 


\section{INTRODUÇÃO}

O sedentarismo é um fator de risco independente para diversas condições médicas cujas morbimortalidade e prevalência têm importância significante para a sociedade e para o sistema de saúde. Doenças cardiovasculares, diabetes, câncere distúrbios neurológicos são enfermidades que podem ser prevenidas, e até tratadas com atividade física, principalmente se essa for associada a uma dieta balanceada (DELEZIE; HANDSCHIN, 2018).

Segundo Pedersen (2019), além de diminuir o risco de doenças crônicodegenerativas, o exercício físico participa da restauração e da manutenção da função cognitiva. Essa participação ficou em evidência quando foram descobertos o Fator Neurotrófico Derivado do Cérebro (BDNF, em inglês) e as miocinas, peptídeoshormônios que atuam em diversos tecidos orgânicos, inclusive no neuronal, produzidos e secretados por células musculares, principalmente quando exercitadas, cujo representante mais relevante, atualmente, é a irisina.

Com base nos pressupostos, o presente estudo tem por objetivo reunir os achados de diferentes pesquisas, a fim de conhecer a relação entre irisina e cognição, esta última entendida de modo amplo, agrupando estudos sobre memória, atenção, tomada de decisões e desempenho.

\section{METODOLOGIA}

Este estudo consistiu na verificação do estado da arte acerca dos estudos sobre irisina e cognição, sendo esta última, especificamente, mensurada a partir de medidas de desempenho e sucesso acadêmico. Ademais, a relação entre esses construtos será analisada em estudos científicos, buscando respostas ao 
questionamento: qual a relação e o poder explicativo da miocina na cognição? Assim, tal estudo serve para embasar ações, tratamentos e outros futuros estudos.

Para demonstrar o estado da arte, foi realizada, inicialmente, uma revisão sistemática. Segundo Rodrigues e Ziegelmann (2010), a revisão sistemática abarca todo o material público sobre o tema delimitado. Ela tem como intuito aproximar o pesquisador da literatura da área, verificando o que já foi produzido acerca do tema da pesquisa. Na revisão sistemática visa-se, ainda, descrever, registrar, analisar e interpretar os dados de pesquisas prévias.

Consideraram-se quatro critérios de inclusão dos trabalhos no presente estudo: (1) ter sido publicado nos últimos seis anos; (2) estar escrito em língua portuguesa ou inglesa; (3) relacionar irisina e cognição; (4) terem por natureza estudos científicos originais.

Assim, a seleção dos artigos foi realizada pelo autor, sendo incluídos todos os artigos publicados entre os anos de 2013 e 2019.

A pesquisa foi realizada nas seguintes bases eletrônicas de dados: PubMed e Medline. Foram utilizadas as estratégias de busca "irisin or myokine and cognition" através da combinação dos descritores (MeSH - medical subject headings). A busca foi realizada entre os dias 01 de junho a 08 de junho de 2019, considerando o período estabelecido.

Os artigos identificados pela estratégia de busca foram avaliados, obedecendo aos critérios de inclusão supracitados. Assim, inicialmente, foi realizada leitura dos títulos e resumos, sendo excluídos aqueles que não contemplassem o objetivo proposto. Posteriormente os textos foram lidos na íntegra, sendo ainda excluídos aqueles que não se enquadrassem nos critérios estabelecidos.

Com o intuito de analisar a revisão sistemática, foram realizadas descrições de natureza bibliométrica. De acordo com Araújo (2006), a bibliometria diz respeito a uma ferramenta de análise estatística que possibilita o mapeamento e a construção de diferentes indicadores de tratamento da informação, possibilitando avaliar o tamanho, o crescimento e a distribuição da bibliografia em certa área de estudo. 


\section{RESULTADOS}

Inicialmente, introduziu-se os descritores através da estratégia de busca "irisin or myokine and cognition" nas bases PubMed e Medline. No total, foram encontrados 762 artigos, sendo 10 do PubMed e 752 do Medline.

Em seguida, realizou-se uma segunda análise, retirando as publicações repetidas, retiveram-se 688 artigos $(90,29 \%)$. O terceiro passo foi realizar uma análise mais aprofundada, considerando-se os títulos e resumos dos artigos e verificando quais se encaixavam nos critérios de inclusão supracitados. Foram retidos 26 artigos $(3,41 \%)$. Dentre os principais motivos para eliminação estiveram: não se tratarem de estudos empíricos; não abordavam a relação entre miocina e cognição. A figura 1 resume o procedimento de seleção dos artigos.

Figura 1. Seleção dos artigos com base na revisão sistemática da literatura sobre irisina e cognição.

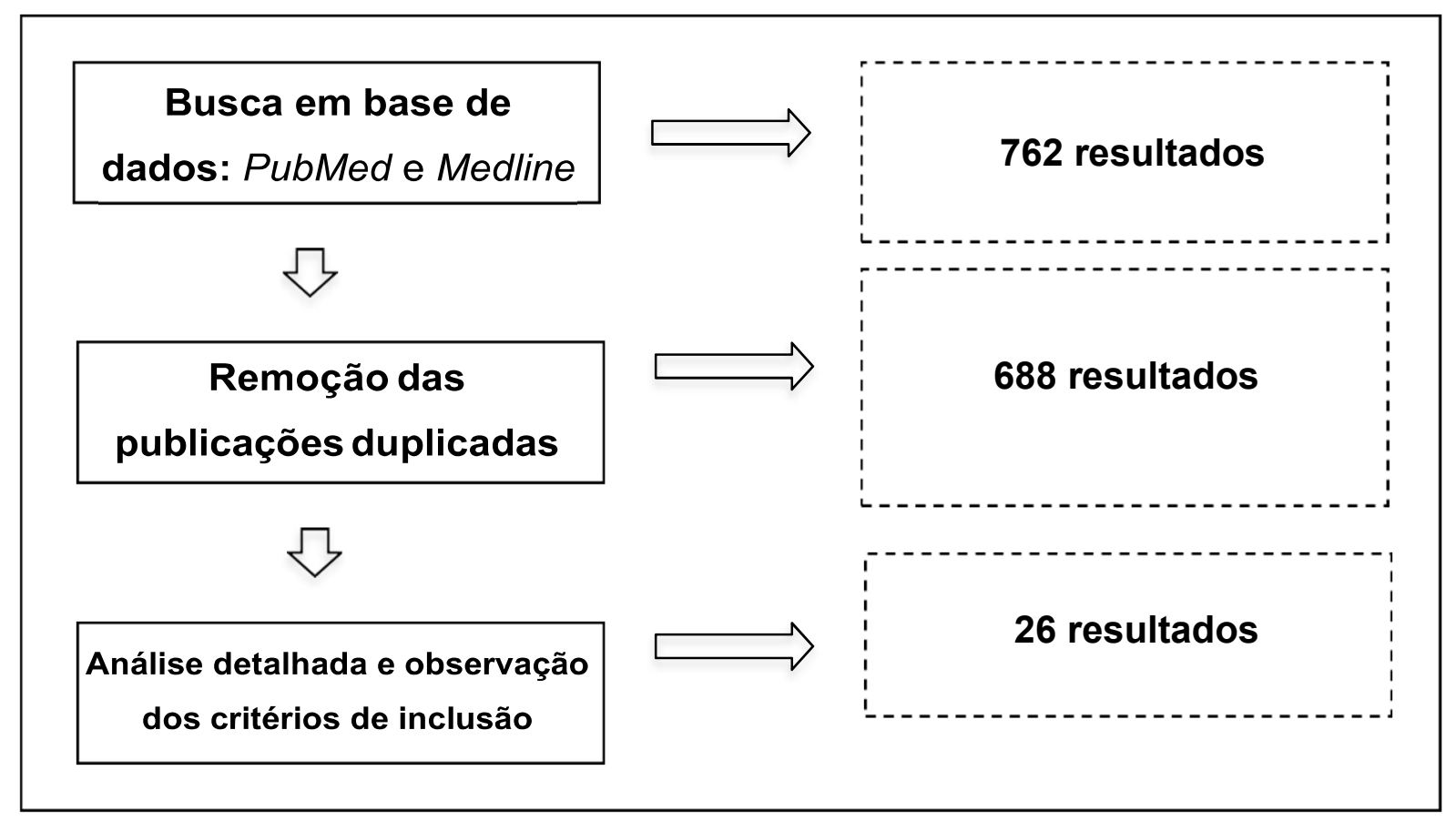

Fonte: autor, 2019. 
Os artigos selecionados foram derivados de um total de 23 revistas, o que indica que não há um predomínio em determinada área, mas que, de forma geral, a comunidade científica tem abordado esse tema com interesse. A revista que apresentou maior predomínio de artigos foi a Cell Metabolism (11,5\%). Destes, a maioria foi do ano de 2018 (26,9\%), sendo o menor quantitativo daqueles publicados em 2014 (3,8\%), o que pode sugerir, novamente, o interesse crescente pela temática. A figura 2 apresenta as publicações por ano.

Figura 2. Ano de publicações dos artigos encontrados na revisão sistemática acerca das temáticas miocinas e cognição.

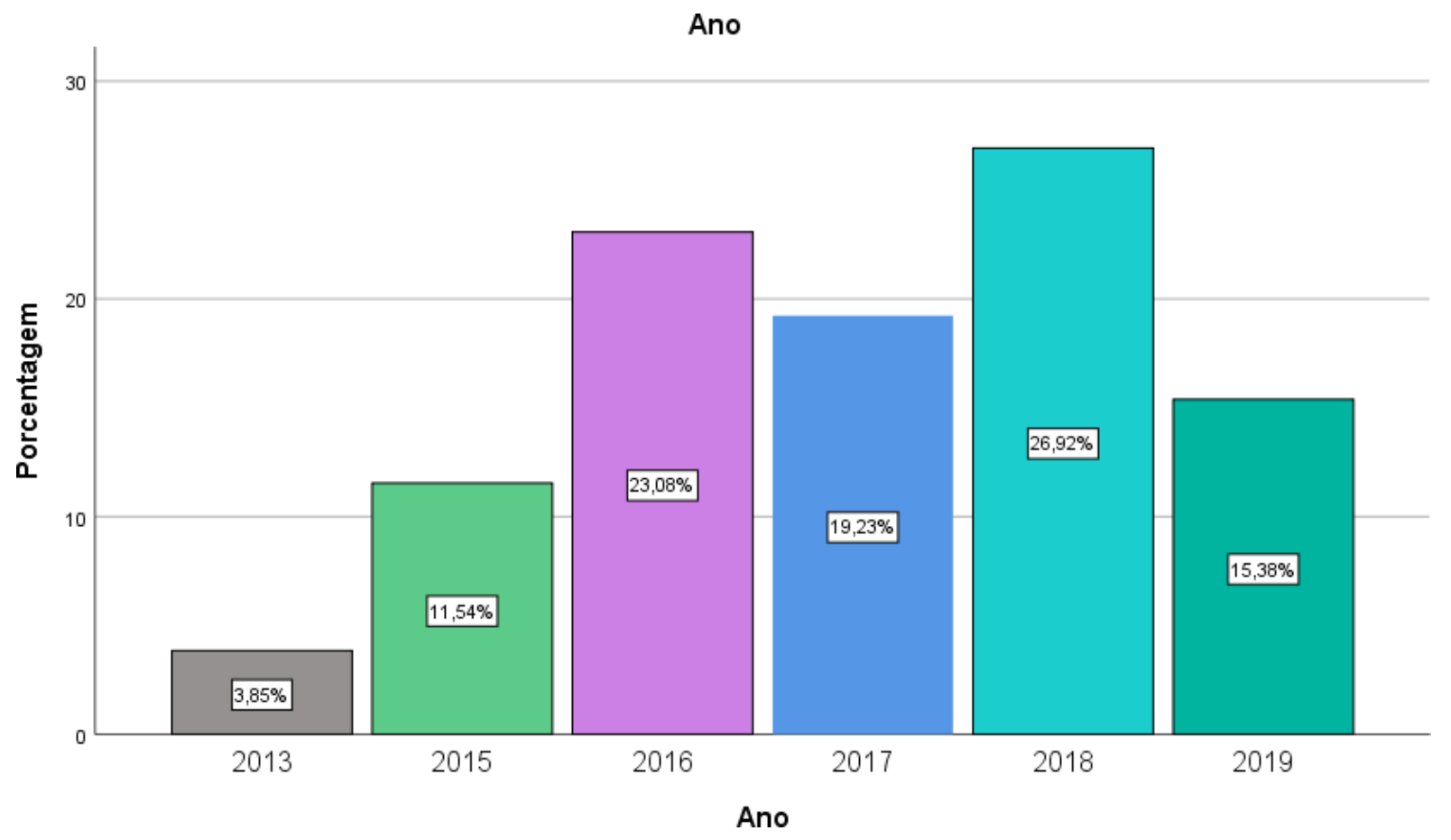

Fonte: autor, 2019.

Também é importante destacar que nenhum artigo foi encontrado em língua portuguesa, mas um deles (COELHO JUNIOR, et al., 2016) foi elaborado no Brasil, por um grupo da Universidade de Campinas. Esses números apontam para a fragilidade no estudo do tema quando ao número de estudos, especialmente no Brasil, tendo em vista a grande defasagem de pesquisas (apenas uma foi encontrada). 
Outro importante elemento abordado foi acerca da natureza do estudo, se de revisão da literatura ou uma pesquisa empírica, de ordem experimental ou correlacional. A figura 3, a seguir, demonstra que a maior parte $(57,69 \%)$ dos estudos encontrados foi de revisão da literatura teórica; os de natureza empírica equivaleram a $42,31 \%$. Destaca-se que não foram encontrados nenhum estudo de natureza metanalítica acerca da relação entre irisina e cognição. Os dados dos estudos empíricos também demonstraram como esse tema ainda carece de mais estudos de natureza experimental, quase experimental e correlacional que venham a explicar melhor essa relação.

Figura 3. Divisão dos estudos da revisão sistemática acerca das temáticas irisina e cognição por tipo de pesquisa.

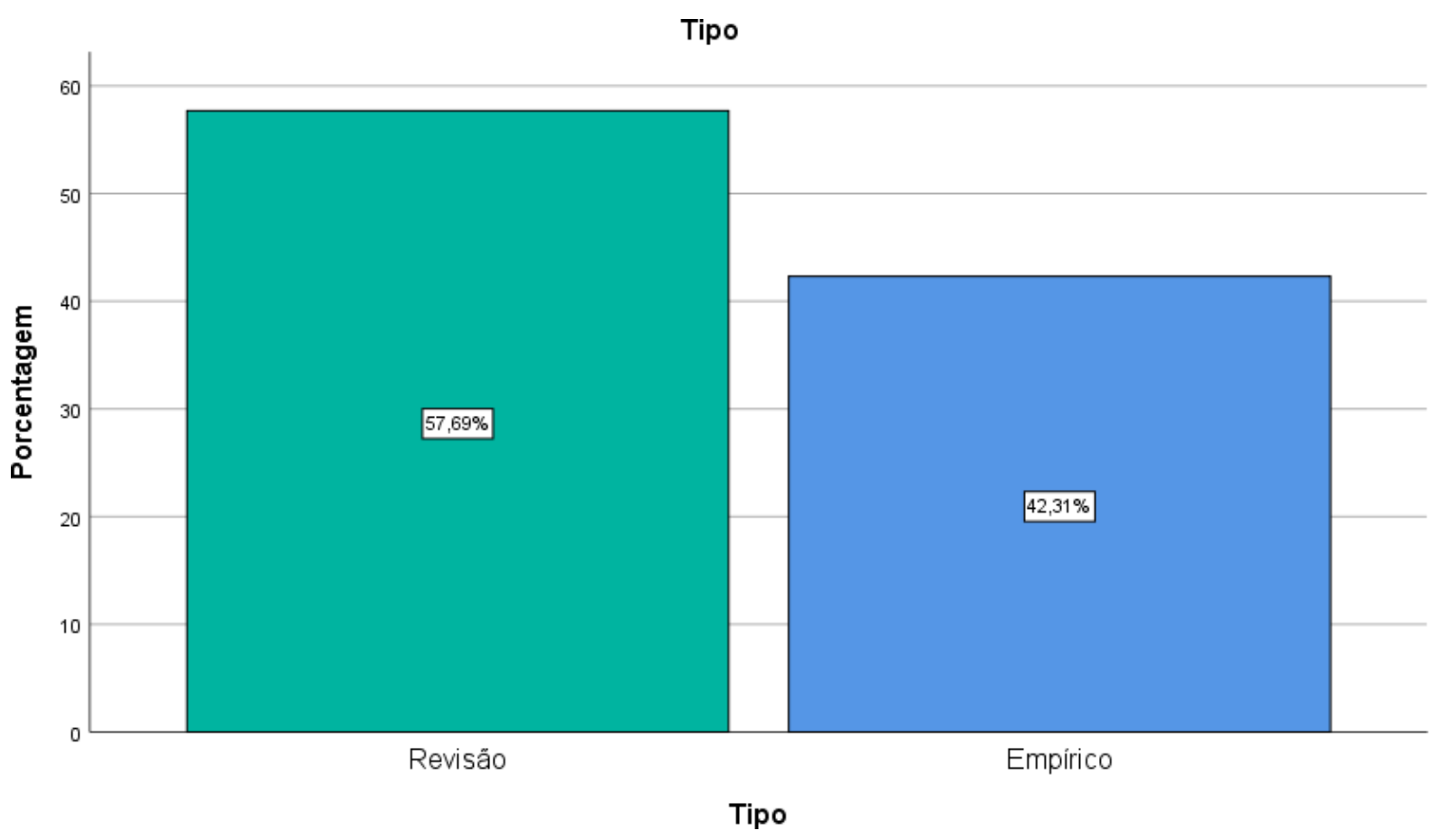

Fonte: autor, 2019.

Em seguida, buscou-se adentrarmais profundamente nas temáticas dos artigos a fim de verificar se, de fato, abordavam propriamente a relação entre irisina e cognição, tendo em vista a observação de que alguns tangenciavam as temáticas, mas sem relacioná-las. Como pode ser verificado na figura 4, apresentada abaixo, $81,82 \%$ dos artigos, de fato, tratavam acerca da relação entre irisina e cognição e 
18,18\%, apesar de falarem de ambos, não os abordava conjuntamente. É importante destacar que esta análise foi feita apenas entre aqueles artigos que eram de natureza empírica $(\mathrm{N}=11)$.

Figura 4. Número de artigos que abordam propriamente a relação entre irisina e cognição.

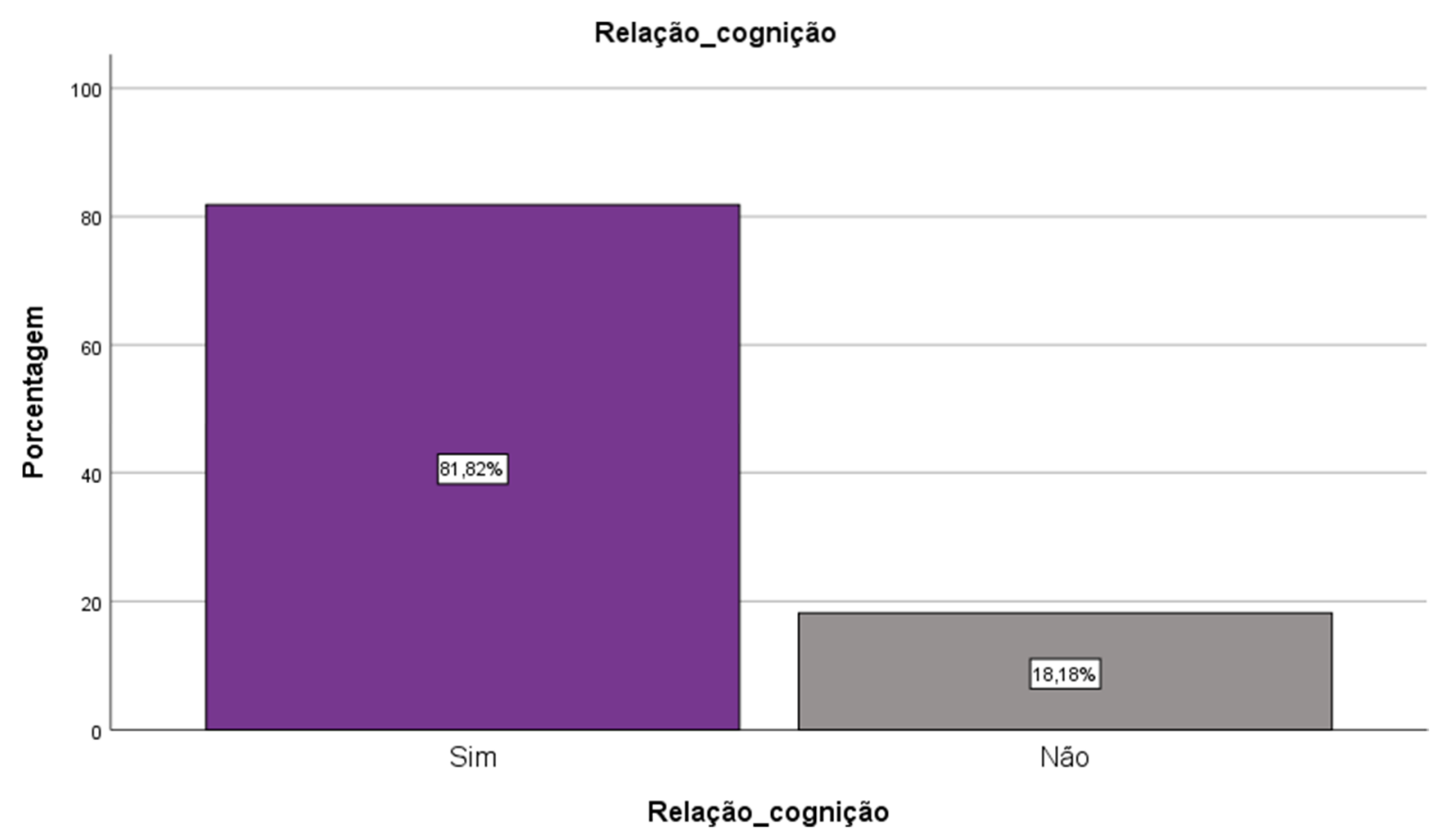

Fonte: autor, 2019.

Considerando esses estudos de natureza empírica, os tamanhos amostrais variaram de 14 sujeitos (GMIAT et al., 2017) a 114 sujeitos (FAGUNDO et al., 2016), com uma média de 51,43 (DP $=34,46)$.

Buscou-se ainda verificar o tipo de amostra mais estudada nas pesquisas empíricas. Foi possível observar (figura 5) que a população de idosos ainda é predominante nos estudos (33,33\%), sendo $11,11 \%$ destes especialmente de idosos com Alzheimer. Também foi possível verificar que 22,22\% dos estudos foram relizados com ratos, o que novamente demonstra que os estudos da área ainda são preliminares. Observou-se ainda que $22,22 \%$ dos estudos foram realizados com pessoas saudáveis em prática de exercícios (BELVIRANLI et al., 2016). 
Figura 5. Características amostrais encontradas nos estudos empíricos que abordam a relação entre irisina e cognição.

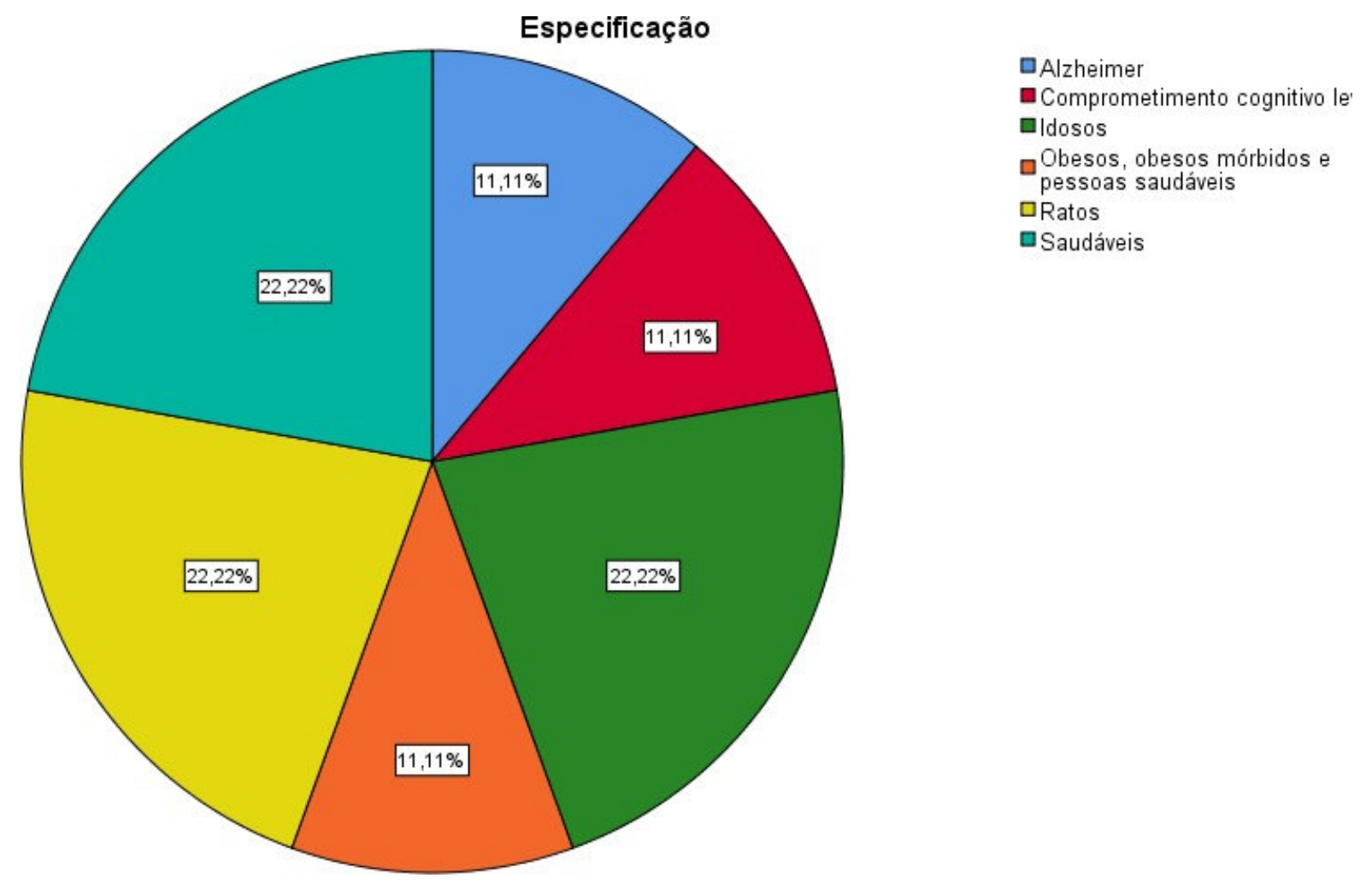

Fonte: autor, 2019.

Outro importante dado avaliado foi sobre o tipo de função executiva trabalhada na avaliação da cognição. Como pode ser observado na figura 6 , as seguintes funções executivas foram verificadas: atenção, fluência verbal, função cognitiva geral, memória de longo prazo, memória de trabalho, memória espacial e teste de inteligência. A memória, portanto, nas suas diferentes dimensões, foi a função mais avaliada (40\%). Ademais, a memória espacial, dentre estas, foi a mais avaliada $(20 \%)$. Também houve um maior predomínio da função cognitiva geral, medida, especialmente, pelo Miniexame do Estado Mental (MEEM). Outras ferramentas de mensuração das funções executivas observadas nos estudos foram: Teste D2 de atenção, Teste de Confecção A \& B, Wisconsin Card Sorting Test, Stroop Cor, Word Test, lowa Gambling Task e Isaac's Set Test of Verbal Fluency (IST). 
Figura 6. Funções executivas trabalhadas na avaliação da cognição.

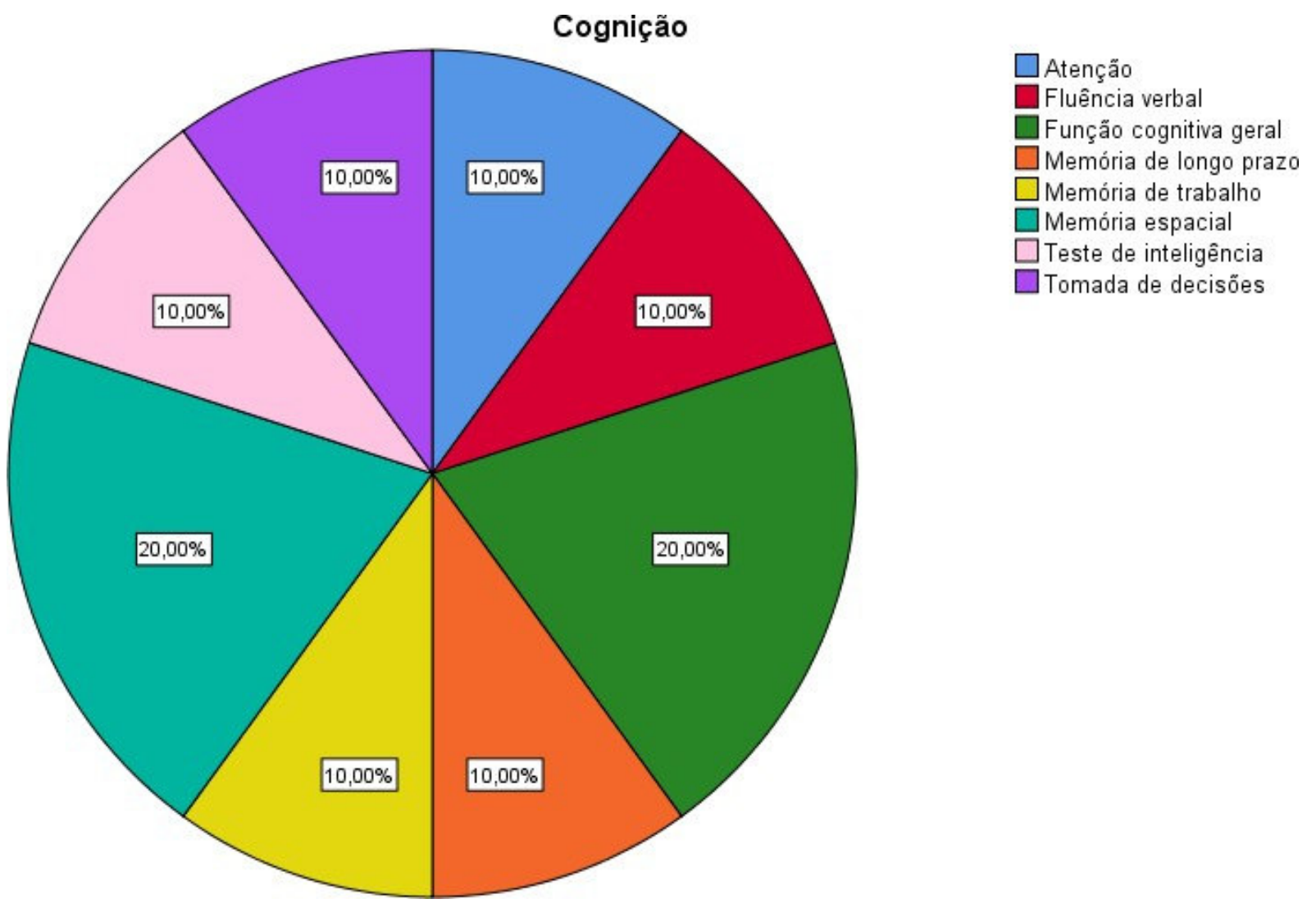

Fonte: autor, 2019.

\section{DISCUSSÃO}

Diante da análise de natureza qualitativa dos estudos observados, foi possível verificar que a descoberta da irisina é recente e os limites do seu efeito ainda estão sendo testados (ZHANG; ZHANG, 2016). Ela é considerada uma nova miocina e seu mecanismo de ação acontece da seguinte forma: ao praticar um determinado exercício, há um aumento da enzima PGC-1alpha no músculoque gera a produção da proteína muscular FNDC5, que é clivada e secretada como irisina. Ademais, o aumento da irisina no sangue induz a expressão do fator neurotrófico derivado do cérebro (Brain-derived neurotrophic factor - BDNF). O BDNF está ativo no hipocampo, 
participando do processo de neurogênese e neuroproteção. Assim, é possível entender que o exercício, cuja irisina funciona como biomarcador, melhora a função cognitiva (VOSS et al., 2019; WRANNet al., 2013).

Na mesma perspectiva, Küster et al. (2017) verificaram em estudo com idosos com risco de demência que o BDNF e a irisina correlacionaram-se positivamente com a cognição global e com a memória episódica. Ademais, os autores também verificaram que eventos de vida de caráter estressante foram associados com BDNF reduzido. Belviranli e Okudan (2018), ainda dentro desses achados, verificaram que a disfunção cognitiva induzida pelo envelhecimento se associa a uma diminuição na expressão do hipocampo de PGC-1alpha, FNDC-5 e BDNF, e que a atividade física pode aprimorar o funcionamento cognitivo via ativação desses genes e proteínas.

Estudos também mostram a relação da irisina na regulação da diferenciação e proliferação neural, neurocomportamento, gasto energético, função cardíaca, aprendizado e memória (ZHANG; ZHANG, 2016). Delezie e Handschin (2018) acrescentam, ainda, a relação com a melhora do sono e do humor.

Jedrychowski (2015) fizeram um estudo para quantificação da irisina humana no plasma. Eles usaram espectrometria de massa com peptídeos de controle enriquecidos com isótopos estáveis. A partir desse método eles observaram que a irisina humana é traduzida, especialmente, do seu códon de início não canônico, circulando a 3.6ng/ml em indivíduos sedentários. Quando os sujeitos são submetidos a treinamento intervalado aeróbico, esse nível aumenta para $\sim 4.3 \mathrm{ng} / \mathrm{ml}$. Assim, os autores concluem que, de fato, a irisina humana existe, circula e é regulada por exercícios físicos.

Belviranli et al. (2016), em seu estudo experimental acerca da relação entre BDNF e habilidades cognitivas, observou uma correlação positiva entre os resultados do teste de função cognitiva e concentrações de BDNF e irisin a $(p<0,05)$. Também observaram uma correlação positiva entre irisina e BDNF $(p<0,05)$ em atletas que fazem treinos de resistência.

Voss et al. (2019) afirmam que, apesar de estar claro que a atividade física aumenta a memória em ratos, os resultados ainda não são claros em humanos. Após análise de revisão eles sugerem o uso de tarefas que sejam mais sensíveis para a 
avaliação dos efeitos da atividade física nas mudanças relacionadas à idade no hipocampo, tal como memória relacional e discriminação mnemônica.

Gmiat et al. (2017) verificaram que, após um circuito de treinamento de alta intensidade, participantes jovens exibiram maior concentração e melhor memória espacial, enquanto em mulheres de meia idade os efeitos observados foram menores. É importante destacar a vitamina $D$ agindo como covariável para mudanças nas funções cognitivas. Esse resultado também foi observado em Gmiat et al. (2018), cuja variável independente foi o treinamento regular de caminhada nórdica, agora em mulheres idosas. Nesse último os autores chegaram à conclusão que houve uma melhora das funções cognitivas após exercício e que não foi o nível de vitamina $D$ que modulou as alterações induzidas pelo exercício, apesar de ter colaborado com as mesmas. Babaei et al. (2017) também abordaram a relação entre irisina e vitamina D, apresentando resultados satisfatórios a uma melhora cognitiva.

Damirchi, Hosseini e Babaei (2017) realizaram um estudo com BDNF e irisina em mulheres diagnosticadas com comprometimento cognitivo leve. Eles observaram uma melhoria significativa na memória de trabalho $(p<0,001)$ e BDNF $(p<0,02)$, quando comparados os grupos experimental e controle. Houve também uma melhora na memória de trabalho $(p<0,01)$ e velocidade de processamento $(p<$ 0,02 ). Os autores destacaram que, mesmo diante dos resultados significativos, os tamanhos de efeito encontrados foram pequenos.

Importante estudo foi realizado por Lourenço et al. (2019), que demonstrou que a superexpressão periférica de FNDC5 / irisina melhora o comprometimen to da memória. Por outro lado, o bloqueio de FNDC5 / irisina periférica ou cerebral atenua as ações neuroprotetoras do exercício físico sobre plasticidade sináptica e memória em camundongos. Em suma, ao demonstrarem que o FNDC5 / irisina é um mediador importante dos efeitos benéficos do exercício em modelos de Alzheimer, os achados dos autores supracitados dão ao FNDC5 / irisina o papel de se opor à falha de sinapse e ao comprometimento da memória no Alzheimer.

Kim et al. (2019), ao revisarem o nível de evidência do efeito da miocina na função cerebral, demonstraram que o nível de evidência associando a mudança na miocina após exercício físico, quanto ao benefício do exercício no cérebro é maior do que acerca da melhora da função neuropsiquiátrica. Mas essa última também é 
importante, associando, por exemplo, a diminuição da fadiga, melhora do humor, das habilidades cognitivas e mobilidade em pacientes com Esclerose Múltipla (SHARIF et al., 2018); redução da depressão e ansiedade (PEDERSEN, 2019; TUKA et al., 2017). Suzuki (2016) também aponta para uma nova miocina que tem apresentado resultados também favoráveis à cognição: a catepsina $B$ (CTSB), que demonstrou uma associação positiva com a memória.

\section{CONCLUSÃO}

Em suma, houve constatação acerca da relevância de estudar a relaçãoentre irisina e cognição, visto que essa miocina, em alguns estudos, foi vinculada a uma melhor função cognitiva, principalmente, quando sua produção é estimulada com o exercício físico. Contudo, o tema deve ser abordado por mais estudos empíricos, para que a associação seja embasada e estabelecida efetivamente.

\section{REFERÊNCIAS BIBLIOGRÁFICAS}

ARAÚJO, C. A. Bibliometria: evolução histórica e questões atuais. Porto Alegre: Em Questão, 2006.

BABAEI, P., et al. Vitamin D is associated with metabotropic but not neurotrophic effects of exercise in ovariectomized rats. Diabetology \& Metabolic Syndrome, v.9, n.91, 2017.

BELVIRANLI, M., OKUDAN, N. Exercise Training Protects Against Aging-Induced Cognitive Dysfunction via Activation of the Hippocampal PGC-1a/FNDC5/BDNF Pathway. NeuroMolecular Medicine, n.20, v.3, 2018, p.386-400.

BELVIRANLI, M., et al. The relationship between brain-derived neurotrophic factor, irisin and cognitive skills of endurance athletes. The Physician and Sportsmedicine, v.44, n.3, 2016, p.290-6.

COELHO JUNIOR, H. J., et al. Inflammatory Mechanisms Associated with Skeletal Muscle Sequelae after Stroke: Role of Physical Exercise Mediators of Inflammation. Mediators of Inflammation, Volume 2016, Article ID 3957958, 2016.

DAMIRCHI, A., HOSSEINI, F., BABAEI, P. Mental Training Enhances Cognitive Function and BDNF More Than Either Physical or Combined Training in Elderly Women With MCl: A SmallScale Study. American Journal of Alzheimer's Disease \& Other Dementias, v.33, n.1, 2017, p.20-29.

DELEZIE, J., HANDSCHIN, C. Endocrine Crosstalk Between Skeletal Muscle and the Brain. 
Frontiers in Neurology, vol.9, art. 698, 2018.

JEDRYCHOWSKI, M. P., et al. Detection and Quantitation of Circulating Human Irisin by Tandem Mass Spectrometry. Cell Metabolism, v.4, n.22, 2015, p.734-740.

FAGUNDO, A. B., et al. Modulation of Irisin and Physical Activity on Executive Functions in Obesity and Morbid obesity. Scientific Reports, v.1, n.6, 2016.

GMIAT, A., et al. Improvement of cognitive functions in response to a regular Nordic walking training in elderly women - A change dependent on the training experience. Experimental Gerontology, v. 104, 2018, p.105-112.

GMIAT, A., et al. The impact of a single bout of high intensity circuit training on myokines' concentrations and cognitive functions in women of different age.

Physiology \& Behavior, v.179, 2017, p.290-297.

$\mathrm{KIM}, \mathrm{S}$., et al. Roles of myokines in exercise-induced improvement of neuropsychiatric function. European Journal of Physiology, v.471, n.3, 2019, p.491-505.

KÜSTER, O. C., et al. Novel Blood-Based Biomarkers of Cognition, Stress, and Physical or Cognitive Training in Older Adults at Risk of Dementia: Preliminary Evidence for a Role of BDNF, Irisin, and the Kynurenine Pathway. Journal of Alzheimer's Disease, v.59, n.3, 2017 , p.1097-1111.

LOURENCO, M. V., et al. Exercise-linked FNDC5/irisin rescues synaptic plasticity and memory defects in Alzheimer's models. Nature Medicine, n.25, 2019, p.165-175.

PEDERSEN, B. K. Physical activity and muscle-brain crosstalk. Nature Reviews Endocrinology, v.15, 2019, p.383-392.

RODRIGUES, C.; ZIEGELMANN, P. Metanálise: Um guia prático. Revista HCPA, Porto Alegre, 2010.

SHARIF, K., et al. Physical activity and autoimmune diseases: Get moving and manage the disease. Autoimmunity Reviews, v.17, n.1, 2018, p.53-72.

SUZUKI, W. A. How Body Affects Brain. Cell Metabolism, v.24, n.2, 2016, p.192-3.

TUKA, V., et al. Physical activity - the Holy Grail of modern medicine? Vnitrni Lekarstvi, v.63, n.10, 2017, p.729-736.

VOSS, M. W., et al. Exercise and Hippocampal Memory Systems. Trends in Cognitive Sciences, v.23, n.4, 2019, p.318-333.

WRANN, C. D., et al. Exercise Induces Hippocampal BDNF through a PGC- 1a/FNDC5 Pathway. Cell Metabolism, v.18, n.5, 2013.

ZHANG, J., ZHANG, W. Can irisin be a linker between physical activity and brain function? Biomolecular Concepts, v.7, n.4, 2016, p.253-8. 